\title{
X.
}

\section{Die Gährungsprobe zum qualitativen Nachweise von Zucker im Harn.}

(Aus dem ehemiscben Laboratorium des pathologischen Institutes zu Berlin.)

\author{
Von Dr. Max Einhorn, \\ Arzt in New-York.
}

Den in der medicinischen Praxis üblichen Methoden zum Nachweis von Zucker liegen folgende Eigenschaften desselben zu Grunde:

1) Metalloxyde in alkalischen Lösungen in Oxydule oder Metalle überzuführen (Reductionsproben).

2) Polarisirtes Licht nach rechts zu drehen (Polarisationsprobe).

3) Unter dem Einfluss von Hefe sich in Alkohol und Kohlensäure zu spalten (Gährungsprobe).

Am meisten im Gebrauch sind die beiden erster Klassen, nehmlich die Reductionsprobe und die Polarisationsprobe, während die Gährungsprobe äusserst selten in Anwendung gezogen wird.

Und doch sind dabei die beiden ersten Arten des Nachweises für das Vorhandensein von Zucker, hauptsächlich in kleineren Mengen, etwa von 0,3 pCt. ab, kaum geeignet. Der Fehler jener beiden Methoden liegt darin, dass weder die Reduction, noch die Rechtsdrehung, noch beide Eigenschaften zusammengenommen ausschliesslich dem Zucker allein im Harn zukommen.

Gerade in den letzten Jahren hat sich der Kreis unseres Wissens in dieser Beziehung vielfach erweitert.

Abgesehen von der, auch dem normalen Harn zukommenden reducirenden Eigenschaft ${ }^{1}$ ), welche allerdings viel schwächer ist,

1) Nach M. Flückiger reducirt der normale menschliche Harn so stark, wie eine 0,15-0,25 procentige Traubenzuckerlösung. M. Flückiger, Zeitschr. f. phys. Chem. Bd. IX. S. 338. 
d. h. langsamer wirkt, als jene des Zuckers, - - und welche nur zum geringeren Theil der Harnsäure und dem Kreatinin zuzaschreiben sein dürfte, zum grösseren Theile noch unbekannten Substanzen, sogenannten Extractivstoffen, angehört, - kommen im pathologischen Harne Substanzen vor, die einerseits ebenso schnell reduciren wie Zucker, andererseits auch solche, die sich nicht indifferent verhalten gegen polarisirtes Licht.

So ist der Harn nach dem Gebrauch von Benzoesäure nach E. Salkowski ${ }^{1}$ ) stark reducirend.

Reducirend und linksdrehend sind sämmtliche Verbindungen der gepaarten Glycuronsäuren, welche erst im letzten Jahrzehnt entdeckt worden sind.

Zuerst gelang es von Mering und Musculus ${ }^{2}$ ) aus dem Harne von Mensehen, denen Chloralhydrat verabreicht wurde, die Urochloralsäure, welche reducirt und linksdreht, rein darzustellen; die Beziehung dieser Säure zu der, damals noch unbekannten, von Jaffe und Schmiedeberg entdeckten Glycuronsäure, wurde erst später nachgewiesen.

$\mathrm{Jaffe}^{3}$ ) zeigte 1878 , dass nach Einverleibung von Orthouitrotoluol im Harn eine stark linksdrehende und Kupferoxyd reducirende Säure erscheine, welche er in Harnstoff and Uronitrotoluolsäure zerlegen konnte; diese Säure vermochte derselbe Autor in zwei Bestandtheile zu zergliedern, in den Orthonitrobenzylalkohol, der sich durch Aether ausfällen liess, während der zweite Bestandtheil, eine Säure, aus der Lösung sich nicht isoliren liess; als wahrscheinliche Formel für diese Säure gab Jaffe $\mathrm{C}_{6} \mathrm{H}_{10} \mathrm{O}_{7}$. an.

Rein dargestell wurde diese Säure zuerst von Schmiedeberg und Meyer ${ }^{4}$ ) und Glycuronsäure benannt; dieselbe reducirt und zeigt rechtsseitige Circumpolarisation; es ist zwar noch nicht constatirt, dass die Glycuronsäure als solche im Haro vorkomme, allein liegt die Möglichkeit immerhin vor.

Nach denselben Autoren wird der Harn reducirend und

1) Zeitschr. f. phys. Chem. Bd. I. S. 25.

2) Berich, d. deutsch. chem. Gesellsch. Bd. VIII. S. 622.

3) Zeitschr. f. phys. Chem. Bd. II. S. 47.

4) Zeitschr. f. phys. Chem. Bd. III. S. 422. 
linksdrehend nach Aufnahme von Campher infolge der Bildung von Camphoglycuronsäuren.

E. Baumann und C. Preusse ${ }^{1}$ ) haben gẹzeigt, dass nach Fütterung von Brombenzol der Harn ausserordentlich stark links dreht und Kupferoxyd reducirt. Der Harn wird ferner linksdrehend nach dem Einverleiben von Dichlorbenzol, Xylol, Cumol, Terpenthinöl, Guajacol, Thymol, Hydrochinon, Resorcin; Brenzkatechin und Orcin, wie dies $K \ddot{1} \mathrm{z}^{2}$ ) angiebt.

Demnach kann eine Reduction im Harn, selbst wenn sie sich schnell vollzieht, nicht immer mit Sicherheit auf Zucker bezogen werden, ebensowenig eine rechtsseitige Circumpolarisation; andererseits darf eine Indifferenz oder selbst eine schwache Jinksdrehung des Polarisationslichtes durch den Harn nicht als absoluter Beweis dafür dienen, dass in demselben kein Zucker vorhanden ist; denn es ist doch gut denkbar, - und kommt in der That vor, - dass sich eine linksdrehende Substanz Oxybuttersäure - neben dem rechtsdrehenden Zucker vorfindet, welche die Rechtsdrehung des letzteren entwẹder aufhebt oder gar noch übertrifft, so dass eine linksdrehung resultirt.

Ferner möchte ich noch an die allerdings vereinzelt dastehenden Fälle von Levulose, also linksdrehendem Zucker, im Harn erinnern, welche K. Zimmer ${ }^{3}$ ) und Seegen ${ }^{4}$ ) beobachtet haben.

Während sich also die Reduction sowohl, wie die rechtsseitige Circumpolarisation im Princip für den Nachweis von Zucker im Harn als ungenügend erwiesen, - ist die Eigenschaft des Zuckers in Lösungen unter dem Einfluss von Hefe zu vergähren, d. h. in Alkohol und Kohlensäure zu zerfallen etwas Specifisches und ihm allein eigen; wir kennen vorläufig noch keine andere Substanz, welche unter denselben Bedingungen einem ähnlichen Zerfalle anheimfiele.

Die Gährungsprobe hat also den grossen Vorzug einer absoluten Richtigkeit der anderen Proben voraus, gleichwohl ist sie bisher wenig cultivirt und angewandt worden.

1) Berich. d. deutsch. chem. Gesellsch. Bd. XII (187). S. 806.

2) E. Külz, P flüg. Arch. Bd. XXVIII. S. 518.

3) K. Zimmer, Deutsche med. Wochenschr. 1876. No. 28.

4) Seegen, Centralbl. f. d. med. Wissensch. 1884, No. 43. 
Die Gährungsprobe ist dabei keineswegs eine der neuesten; schon Home kannte die Eigenschaft des diabetischen Harns mit Hefe zu vergähren.

Mehr als zum qualitativen Nachweis ist die Gährungsprobe zur quantitativen Bestimmung des Zuckers verwerthet worden. Die Methode, welche übrigens ziemlich alten Datums ist, besteht darin, dass man eine bestimmte Quantität Harn vor und nach der Vergährung wägt und aus dem Gewichtsverlust, welcher von der frei gewordenen Kohlensäure herrïhrt, die Menge des zerfallenen Zuckers berechnet. Diese Art der quantitativen Zuckerbestimmung erfordert viel Mühe und Zeit und kann ausserdem nur von Chemikern ausgeführt werden.

William Roberts ${ }^{1}$ ), der die Schwierigkeiten der eben angeführten Methode kannte, zugleich aber einsah, von welch' hohem Werthe es wäre, einen derartigen quantitativen Bestimmungsmodus zu habeu, den jeder practische Arzt leicht in seine Wohnung ausführen könnte, - kam auf den sinnreichen Gedanken, die bei der Gährung statthabende Verringerung des specifischen Gewichtes des diabetischen Harns zu diesem. Behufe zu benutzen. Roberts beschreibt sein Verfahren wie folgt: „Etwa 4 Unzen diabetischen Harns werden in eine Zwölfunzenflasche gegossen, und ein Stück deutscher Hefe von etwa Wallnussgrösse oder so wird zugesetzt. Die Flasche wird dann lose verkorkt oder mit einer Glasplatte bedeckt und auf den Kaminsims oder eine andere warme Stelle behufs der Gährung hingestellt. Drei oder vier Unzen desselben Urins werden in gleicher Weise, aber ohne Hefe, in eine zweite Flasche gethan, fest verkorkt und neben die gährende Flasche hingestellt.

"Die Gährung beginnt bald und schreitet mit solcher Schnelligkeit vor, dass in 24 Stunden nicht nur dieser Prozess zu Ende ist, sondern auch der Schaum und die Hefe von der Oberfläche verschwunden sind, und der Urin, wenn auch noch etwas trübe, doch bereits geeignet ist, für die zweite Stufe des Verfahrens, - nehmlich die Dichtigkeitsbestimmung. Es ist jedoch rathsam, die Flaschen ein paar Stunden zuvor vom Kaminsims zu entfernen, so dass sich der Inhalt derselben bis auf die allgemeine Zimmertemperatur abgekühlt hat.

1) Wm. Roberts; Edinbourgh med. Journ. Oct. 1861. p. 326. 
"Das specifische Gewicht beider Producte wird sodann getrennt auf die gewöhnliche Weise beobachtet, - indem man den Trin in ein cylindrisches Becherglas hineingiesst und das Urometer hineinsenkt. . . . „Die Differenz beider specifischen Gewichte mit 0,23 multiplicirt gleicht dem Procentgehalt des Harns an Zucker."

Diese Zahl hatte Roberts empirisch gefunden.

Die Methode ist einfach und leicht auszuführen und R. empfiehlt sie warm den Aerzten und möchte sie mit Hinsicht auf ihre practische Brauchbarkeit bei den Aerzten die "klinische" Methode genannt wissen. Er sagt: "I would suggest that this would be called a clinical method of estimating sugar, in order to distinguish it from another and older fermentation process, the principle of which is to determine the weight of carbonic acid evolved in fermentation, and there from to calculate the quantity of sugar broken up."

Manassein ') hat das Verdienst zehn Jahre später darauf aufmerksam gemacht zu haben, dass die Roberts'sche Methode der quantitativen Zuckerbestimmung nicht die verdiente Beachtung gefunden hat, und empfiehlt sie aufs Neue, nachdem er sie selber geprüft und sich von ihrer practischen Brauchbarkeit überzeugt hatte.

Antweiler und Breidenbend ${ }^{2}$ ) suchten die Roberts'sche Methode zu vereinfachen, indem sie die Gährungsdauer abkürzen wollten, und geben an, dies erreicht zu haben durch einen Zusatz von 2,0 weinsaurem Kalinatron und 2,0 phosphorsaurem Kali auf $100 \mathrm{ccm}$ Harn; -- die Hefequantität, die sie angeben, stimmt mit der Roberts'schen Vorschrift ziemlich überein; sie nehmen 10,0:100,0, Roberts $9,6: 100,0$.

Ob der Salzzusatz wirklich den Gährungsprozess so sehr beschleunigt, dass derselbe nach 2-3 Stunden zu Ende ist, und die Roberts'sche Methode wesentlich vereinfacht, möchten wir dahingestellt sein lassen.

Den qualitativen Nachweis von Zucker durch die Gährungsprobe anlangend, so findet man in der Literatur äusserst wenig

1) Manassein, Centralbl. f. d. med. Wissensch. 1872. S. 551.

2) Antweiler und Breidenbend, Pfläg. Arch. Bd. 28. S. 179. 


\section{8}

darüber; höchstens wird sie ab und zu angeführt, da, wo es sich darum handelt zu beweisen, dass eine reducirende Substanz im Harn Zucker gewesen sein muss; so empfiehlt 0 . Rosenbach ${ }^{1}$ ) eine Quantität des betreffenden Harns mit Hefe zu versetzen und nach etwa 24 Stunden seine Reductionsfähigkeit von Neuem zu prüfen; war die reducirende Substanz Zucker; so muss jetzt nach der Vergährung die Reductionsfähigkeit des Harns verringert resp. verschwunden sein.

Bei der Wichtigkeit eines sicheren Nachweises von lkJeinen Mengen Zucker im Harn, haben wir die vielerorts etwas vernachlässigte Gährungsprobe auf Anrathen des Herrn Professor Dr. E. Salkowski zum Gegenstande unserer Untersuchung gemacht. Zunächst galt es festzustellen, wie weit die Empfindlichkeit dieser Probe gehe und dann wie sie gesteigert werden könne.

Die Gährungsprobe beruht, wie schon erwähnt, auf der Eigenschaft sämmtlicher Zuckerarten in Lösungen unter dem Einfluss von Hefe bei geeigneter Temperatur in Alkohol und Kohlensäure zu zerfallen; und zwar giebt ein Molecül Zucker zwei Molecüle Alkohol und zwei Molecüle Kohlensäure nach der Gleichung:

$$
\mathrm{C}_{6} \mathrm{H}_{12} \mathrm{O}_{6}=2 \mathrm{C}_{2} \mathrm{H}_{6} \mathrm{O}+2 \mathrm{CO}_{2} \text {. }
$$

Die bei der. Gährung frei werdende Kohlensäure dient uns zur Erkennung der stattgehabten Reaction.

Zur Anstellung der Probe bedient man sich am besten des im Salkowski-Leube'schen ${ }^{2}$ ) Buche "die Lehre vom Harn" angegebenen und abgebildeten Gährungsröhrchen. Dasselbe ist aus Glas angefertigt und setzt sich aus vier Theilen zusammen: 1) einem kugelförmigen oder ovalen, mit kleiner Oeffnung versehenen Reservoir, 2) einem etwa doppelt so langen, oben. rnnd endigenden cylindrischen Rohr, 3) einer etwas gekrümmten' schmalen Verbindungsröhre, 4) einem Fusse.

\section{Methode.}

Man schüttelt die zu untersuchende Flüssigkeit (etwa $10 \mathrm{ccm}$ ) in einem Reagensgläschen mit käuflicher. Presshefe, welche nicht

1) O. Rosenbach, Breslauer ärztliche Zeitschr. 1884. No. 19.

2) Salkowski und Leube, Die Lehre vom Harn. S. 223. 
schlecht riechen darf und die Consistenz von etwas festem Teig haben muss, gut durch, bis die Hefe nicht mehr in Stücken daliegt, sondern sich gleichmässig vertheilt hat und füllt damit das Reservoir, d. h. den kugeligen Theil des Gührungsröhrchen; durch einiges Neigen gelingt es leicht, das cylindrische Rohr ganz mit der Flüssigkeit zu füllen und sämmtliche Luft aus demselben hinauszutreiben. Ist dieses geschehen, so wird das Gährungsröhrchen wieder auf den Fuss gestellt; die Flüssigkeit bleibt im Cylinder und in der Verbindungsröhre stehen und fliesst nicht in das Reservoir zurück, weil die sich dort vorfindende mit der Atmosphäre communicirende Luft einen Druck auf das Fluidum ausübt und dasselbe nicht weiter vordringen lässt.

Nun wird etwas Quecksilber in das Gährungsröhrchen hineingegossen; dasselbe fällt nach unten in die Verbindungsröhre hinein, füllt dieselbe aus und drängt die sich dort vorfindende Flüssigkeit in das Reservoir hinein.

Jetzt ist das cylindrische Rohr mit der zu untersuchenden Flüssigkeit gefüllt and von der atmosphärischen Luft durch das Quecksilber abgeschlossen.

Dieses Gährungsröhrchen wird, nachdem es so gefüllt ist, in einer Temperatur von etwa $30^{\circ}-33^{\circ}$ für $20-24$ Stunden sich selbst überlassen.

Tritt die alkoholische Gährung ein, so muss die frei werdende Kohlensäure den obersten Theil des cylindrischen Rohres einnehmen und die Flüssigkeit zurückdrängen.

Der weite Abstand des Flüssigkeitsniveau von der Spitze des cylindrischen Rohres dient als Beweis der eingetretenen Reaction. Dass das Gas, welches sich im oberen Theile des cylindrischen Rohres angesammelt hat, wirklich Kohlensäure ist, davon kann man sich dadurch überzeugen, dass man das Reservoir mit Natronlauge vollgiesst, darauf mit dem Finger die Oeffnung schliesst und einige Male die Flüssigkeit durchschüttelt; die Kohlensäure von der Natronlauge absorbirt, der Gasraum verschwindet, der Finger wird in Folge dessen kräftig angesogen, und nur mit Mühe vermag man denselben von der Oeffnung abzuheben.

Zur Anstellung der Gährungsprobe sind, wie l. c. S. 224 ausgeführt wird, 3 Gährungsröhrchen nothwendig: das eine A 
wird mit normalem zuckerfreien Harn und Hefe gefüllt; das zweite B mit dem Untersuchungsharn; das dritte $\mathrm{C}$ mit einem mit Zucker versetzten Harn gefüllt. (C dient nur dazu, um sich von der Wirksamkeit der Hefe zu überzeugen.)

Ist bei $B$ der obere Theil des cylindrischen Rohres von Flüssigkeit leer, während bei A nur eine kleine Gasblase an der Spitze vorhanden ist, so enthält B Zucker.

Ist dies nicht der Fall, d. h. ist in B nur eine Gasblase da von derselben Beschaffenheit wie bei $A$, so könnten wir mit Sicherheit sagen, dass $B$ keinen Zucker enthält, sobald $C$ jenen Gasraum aufweist und wir also sehen, dass die Hefe wirksam war. -

Nach diesen vorausgeschickten Erörterungen gehen wir zu unseren speciellen Untersuchungen über.

Zunächst war die Frage zu beantworten, wie weit geht die Empfindlichkeit der Gährungsreaction überhaupt, und wie in der uns am meisten interessirenden Flïssigkeit, nehmlich im Harn?

Um dieses feststellen zu können, bedienten wir uns mit einer bestimmten Quantität Traubenzuckers versetzter Lösungen von Wasser und Harn.

Die Gährungsröhrchen, die wir zur Verfügung hatten, waren alle ziemlich gleich gross; der Inhalt des cylindrischen Rohres betrug etwa $5 \mathrm{ccm}$.

Im mit Bestimmtheit zu wissen, wie der Zucker gewirkt hat, mussten wir stets Controlproben von derselben Flüssigkeit ohne Zucker anstellen.

Unsere Versuchsanordnung in dieser' Hinsicht war nun folgende:

3. Den 18. Juni 1885. Harn, spee. Gew. 1016.
$\left.A^{1}\right)$. Wasser + Hefe
B. Harn + Hefe
C. Wasser mit $\frac{1}{2} \mathrm{pC}$. Gehalt an Zucker + Hefe
D. Harn

Nach 24 Stunden bei A an der Spitze des cylindrischen Robres ein

1) Die Buchstaben A, B, C u. s. f. bezeichnen bei uns überall die Gäbrungsröbrchen, welche mit den betreffenden Flüssigkeiten versehen waren. 


\section{1}

linsengrosses Gasbläschen; bei $B$ ebendasselbe, aber grösser; bei $C \frac{1}{4}$ des Robres vom Gas eingenommen; bei $\mathrm{D} \frac{1}{3}$.

2. Den 18. Mai 1885. Harn, spec. Gew, 1022 .
A. Wasser + Hefe.
B. Harn + Hefe.
C. Wasser mit $\frac{1}{4}$ pCt. Gehalt an Zucker + Hefe
D. Harn

Nach 24 Stunden bei A und B dieselben Erscheinungen, wie gestern; bei $C$ ist $\frac{1}{8}$ des Rohres vom Gas gefülit, bei D etwa $\frac{1}{5}$.

3. Den 19. Mai 1885. Harn, spec. Gew. 1015.
A. Wasser + Hefe.
B. Harn + Hefe.
C. Wasser mit $\frac{1}{10}$ pCt. Gehalt an Zucker + Hefe.
D. Harn

Nach 24 Stunden bei A wie in beiden vorigen Malen; bei B etwas grösser als in 1 und 2; bei $\mathrm{C}$ ist etwa $\frac{1}{20}$ des Rohres vom Gas angefülllt; bei $\mathrm{D} \frac{1}{15}$.

In der ganzen vorgeführten Versuchsreihe suchten wir uns davon zu überzeugen, dass die entstandenen Gasvolumina Kohlensäure waren, indem wir nach dem, oben angegebenen, Verfahren Natronlauge zusetzten und durchschüttelten; die Flüssigkeit stieg sofort in die Höhe und füllte das cylindrische Rohr aus, es blieb aber stets an der Spitze immer ein Glasbläschen von reichlich Linsengrösse zurück. - Auch zu den zuckerfreien Controlproben von Wasser und Harn mit Hefe setzten wir Natronlauge zu und fanden, dass die Ansaugung des Fingers eine kaum merkliche war, konnten aber gleichzeitig deutlich erkennen, dass die betreffenden Bläschen, wenn auch um ein Geringes, sich doch immerhin verkleinert haben. -

Aus diesen Versuchen geht hervor:

1) dass die Hefe an und für sich mit zuckerfreiem Wasser oder Harn versetzt im Gährungsröhrchen nach einiger Zeit eine Gasblase an der Spitze des Cylinders zur Erscheinung bringt, welche nach 24 Stunden im Wasser etwa linsengross, im Harn jedoch beträchtlich grösser ist;

2) dass bei einem Gehalt der Flüssigkeit an Zucker das Gasvolumen bedeutend vergrössert ist und zwar nimmt das Gasvolumen der Menge des Zuckers entsprechend ziemlich proportional zu. 
3) Der Stand der Flüssigkeit war im zuckerhaltigen Harn stets ein niedrigerer, als im entsprechenden zuckerhaltigen Wasser, d. h. die Gasvolumina im Harn grösser, als die im Wasser; daraus geht hervor, dass die Gährung im Harn besser und vollständiger vou Statten geht, als im Wasser.

4) Wir haben bei $\frac{1}{10} \mathrm{pCt}$. Zucker zwar noch die erwartete Reaction bekommen, indem im Harn $\frac{1}{15}$ des cylindrischen Raumes vom Gas eingenommen war, während beim zuckerfreien Harn nur die convexe Abrundung des cylindrischen Theils von der Flüssigkeit leer war, - gleichviel mussten wir uns sagen, dass wir hier auch an der Grenze angelangt seien. Denn bei noch kleineren Mengen von Zucker würde es wohl schwer werden, den nur kleinen Gasraum von jenem normalen zu unterscheiden. Ausserdem war die Gasblase beim zuckerfreien Harn nicht constant gleich gross, sondern unterlag einigen Schwankungen.

Somit würde man $\frac{1}{10}$ pCt. Zacker im Harn vermittelst der Gährungsprobe ohne Weiteres nachweisen können.

Wir kommen nunmehr zum zweiten Theil der von uns unternommenen Arbeit, nehmlich wodurch lässt sich die Empfindlichkeit der Reaction steigern?

Ueberlegen wir, dass die deutliche Erkennung der stattgehabten Zuckerreaction von der grossen Differenz des Gasvolums im Gährungsröhrchen eines zuckerhaltigen und zuckerfreien Harns abhängt, - so sind offenbar zur Verstärkung der Reactionsempfindlichkeit zwei Wege zu betreten:

1) nach Mitteln zu suchen, die den Gährungsprozess begünstigen und dadurch ein grösseres Gasvolumen im zuckerhaltigen Harn bilden, so dass dann vielleicht noch kleinere Quantitäten von Zucker, als $\frac{1}{10}$ pCt. von zuckerfreiem Harn sich unterscheiden liessen.

2) Die Gasblase, d. h. das Gasvolum des zuckerfreien Harns möglichst zu verkleinern, denn es ist klar, dass auch dadurch die Differenz grösser werden müsste und also eine deutlichere Erkennung ermöglichte.

Wir betreten nun zuerst den ersten Weg und untersuchen im Folgenden, wie sich der Zusatz einiger Substanzen, die den Gährungsprozess begünstigen sollen, im Harn verhält. Denn es 
ist leicht einzusehen, dass der Harn, eine so complicirte Flüssigkeit, sich anders in dieser Beziehung verhalten könnte, als irgend ein anderes indifferentes Mittel.

Wir versuchten es zuerst mit der von A. Mayer empfohlenen Weinsäure. Wir stellten eine 1 procentige Weinsäurelösung her und fügten eine bestimmte Anzahl von Tropfen immer zu $10 \mathrm{ccm}$ Harn zu.

Folgende Versuche wurden nun in der Richtung ausgeführt:

1. Den 20. Mai 1885. Harn, spec. Gew. 1019.

A. Harn + Hefe

B. Harn mit $\frac{1}{4}$ pCt. Gehalt an Zucker + Hefe

C. - - - - - - - +1 Tropfen Weinsäurelösung

D. - - - - - - - + 20

Nach 20 Stunden: Bei A die bereits oben beschriebene Gasblase; B etwa $\frac{1}{6}$ leer von Flüssigkeit, $\mathrm{C}$ etwas mèh, D nur $\frac{1}{10}$.

2. Den 22. Mai 1885. Harn, spec. Gew. 1014.

A. Harn + Hefe +1 Tropfen Weinsäurelösung

B. $\quad-\quad-\quad+20$

C. Harn mit $\frac{1}{4}$ pCt. Gehalt an Zucker + Hefe

D. - - - - - - - - $\quad+1$ Tropfen Weinsäurelösung

E. - - - - - - -+10 -

Nach 24 Stunden bei A und B eine Gasblase von derselben Beschaffenheit, wie bei zuckerfreiem Harn ohne Weinsäurezusatz, bei C und D etwa $\frac{1}{6}$ von der Flüssigkeit leer, bei $\mathrm{E}$ nur $\frac{1}{8}$.

3. Den 27. Mai 1885. Harn, spec. Gew. 1021.

A. Harn mit $\frac{1}{4}$ pCt. Gehalt an Zucker + Hefe

B. - $\quad-\quad-\quad-\quad-\quad-\quad+1$ Tropfen Weinsüurelösing

C. - - - - - - - $-4+5-$

Am folgenden Tage bei $A \frac{1}{1 \frac{1}{2}}, B \frac{1}{10}, \mathrm{C} \frac{1}{10}$ von Flüssigkeit leer.

4. Den 28. Mai 1885. Harn, spec. Gew. 1017.

A. Harn mit $1 \mathrm{pCt}$. Gehalt an Zucker + Hefe

B. - - - - - - $\quad-\quad+1$ Tropfen Weinsäurelösung

D. $-\overline{\text { Nach }} 24$ Stunden bei $\mathrm{A}, \mathrm{B}$ und $\mathrm{C} \frac{1}{5}$ von Flüssigkeit leer.

Das wesentlicbe Ergebniss dieser Versuchsreihe ist, dass die Weinsäure in ganz kleinen Quantitäten ( $1-5$ Tropfen 1 procentige Weinsäure $: 10 \mathrm{ccm}$ ) der Gährung nicht schadet, aber auch nicht viel nützt, dass sie dagegen in grösseren Mengen die Gährung im Harn sogar erheblich beeinträchtigt. 
Wir fürchten den Leser zu ermüden, wollten wir alle Versuche, welche wir in dieser Hinsicht gemacht haben, einzeln anführen. Es genüge zu sagen, dass wir es noch mit dem für den Harn von Antweiler und Breidenbend ${ }^{1}$ ) empfohlenen Salzzusatz (2 g Kalibiphosphat, $2 \mathrm{~g}$ Kal. Natr. tart. : $100 \mathrm{ccm}$ ) versucht haben, dass wir uns aber von dessen günstigen Einfluss für unsere Zwecke gleichfalls nicht haben überzeugen können.

Etwas besser ging es uns mit dem Zusatz von Pepton; es erwies sich nehmlich, dass 1-2 Tropfen einer $2 \frac{1}{2}$ procentigen Peptonlösung auf $10 \mathrm{ccm}$ Harn die Gährung erheblich begünstigten, 5 Tropfen dagegen schon schadeten.

Allein gerade da, wo es darauf ankam, die Gährung zu begünstigen, um ein möglichst grosses, von zuckerfreiem Harn leicht unterscheidbares Gasvolumen zu bekommen, und weswegen wir auch alle diese Versuche überhaupt anstellten, - nehmlich bei kleinen Quantitäten von Zucker, wie etwa $\frac{1}{10}$ pCt. und darunter, erwies sich auch dieses Mittel ganz ohne Einfluss auf die Gährung.

Wir waren also auf diese Weise genöthigt, den ersten von

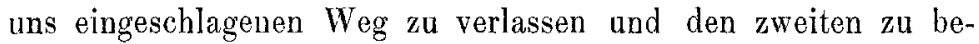
treten, nehmlich die Gasblase, d. h. das Gasvolumen, das sich im zuckerfreien, mit Hefe versetzten Harn bildet, möglichst zu verringern.

Sollte es uns beispielsweise gelingen die Gasblase des normalen Harns ganz zum Verschwinden zu bringen, dann wäre es ja möglich selbst Spuren von Zucker durch ein auftretendes Gasbläschen zu erkennen.

Um irgend welche Aussicht auf Erfolg bei der Betretung des von uns vorgeschriebenen Weges zu haben, war es zunächst nöthig über das Wesen und die Natur der sich im zuckerfreien Wasser oder Harn mit Hefe bildenden Gasblase ins Klare zu kommen.

Woraus besteht diese Gasblase und wovon rührt sie her?

Wir haben bereits oben erwähnt, dass sich diese Gasblasen nach Zusatz von Natronlauge verkleinerten; also müssen sie zu-

I) Pfläg. Arch. Bd. 28. S. 179. 
nächst etwas Kohlensäure enthalten. Ueber die Natur des übrigen und grösseren Theiles der Gasblase vermochten wir keine Gewissheit zu erlangen, und waren wir nur auf Vermuthungen angewiesen.

Es war zunächst denkbar, dass die Gasblase von den im Harn absorbirten Gasen herrührt. Dafür sprach, dass sie bei Anwendung von destillirtem Wasser nie so gross war, wie beim Harn.

Um diese Annahme zu prüfen, war es nur nöthig, zuckerfreie Flüssigkeiten, die von Luft und Kohlensäure frei waren, mit Hefe zu versetzen und abzuwarten, ob sich auch hier die Gasblase bildet oder nicht. Ist unsere Annahme richtig, so dürften wir keine Gasblase erhalten, - ist sie aber falsch, so müssten wir eine Gasblase von derselben Beschaffenheit erhalten, wie sie in unseren früheren Versuchen stets auftrat.

Am leichtesten erschien es uns durch Kochen der Flüssigkeit die absorbirten Gase żu vertreiben.

Wir kochten zu diesem Behufe Wasser und $100 \mathrm{ccm}$ Harn eine halbe Stunde lang, brachten dann den Harn, um wieder dieselbe Concentration zu haben, indem wir ihn mit einem Theil des gekochten Wassers verdünnten, auf das ursprüngliche Volumen zurück, versetzten dann $10 \mathrm{ccm}$ gekochten Wassers und Harns mit Hefe und füllten damit zwei Gährungsröhrchen: zwei Controlgährungsröhrchen mit nicht gekochtem Wasser und Harn wurden gleichzeitig hingestellt, wie dies folgender Versuch zeigt:

Den 2. Juni 1885.
A. Wasser ungekocht + Hefe
B. Wasser gekocht t Hefe
C. Harn ungekocht + Hefe
D. Harn gekocht + Hefe.

Nach 24 Stunden bei $\mathrm{A}$ und $\mathrm{C}$ die gewöhnlichen Erscheinungen; bei $\mathrm{B}$ ein stecknadelkopfgrosses Bläschen, bei D ein um ein Geringes grösseres ebensolches Bläschen.

Ist nun diese Annahme richtig oder falsch? - Die grosse, beinahe die ganze Kuppel des Gährungsröhrchens ausfüllende Gasblase im Harn ist zwar verschwunden, allein es ist noch ein stecknadelkopfgrosses Bläschen zurückgeblieben, ferner war dasselbe im Harn etwas grösser als im Wasser. Das Alles liess uns vermuthen, dass unsere erste Annahme nicht richtig war; 
denn wir mussten doch dieses Bläschen, wenn es wirklich Luft ist, als von der, während des Abkühlens der Flüssigkeiten wieder absorbirten Luft herrïhrend betrachten und dann, wäre es nicht klar, warum der Harn, der doch in derselben Zeit gewiss nicht mehr absorbirt hat, als das Wasser, ein grösseres Bläschen hervorbringt.

Der Leser bemerkt wohl, dass wir hier den Punkt bereits gefunden haben, auf den es uns hauptsächlich ankam - und dessentwillen wir die ganze Frage über die Natur und Entstehung der Gasblase aufgeworfen haben, - nehmlich die Gasblase zu einem kleinen Gasbläschen herabzusetzen, - gleichwohl möchten wir noch zuerst, bevor wir zu unserem speciellen Thema zurückkehren, die bereits aufgeworfene Frage nach Kräften erledigen.

Unsere Befürchtung, dass die erste Annahme keine richtige war, hat sich bestätigt, als wir die andere Möglichkeit untersuchten, nehmlich ob nicht die Gasblase ein Erzeugniss der Hefe selbst sei. Es ist augenscheinlich, dass viel Hefe dann eine grössere Gasblase liefern müsste, als wenig Hefe.

Wir stellten daher zu diesem Behufe folgenden Versuch an:

Den 11. Juni 1885.
A. Wasser +1 Theil Hefe ${ }^{1}$ )
B. Wasser +20 Theilen Hefe
C. Harn +1 Theil Hefe
D. Harn +20 Theilen Hefe.

Nach 24 Stunden bei A ein kleinstecknadelkopfgrosses Bläschen, bei $B$ ist die ganze Kuppel des cylindrisehen Rohres vom Gase eingenommen; bei $\mathrm{C}$ ein grossstecknadelkopfgrosses Bläschen, bei $\mathrm{D}$ beginnt der Gasraum bereits $2-3 \mathrm{~mm}$ vor der Kuppel.

War somit erwiesen, dass die Gasblasen in zuckerfreien Flüssigkeiten ein Product der Hefe sind, so fragten wir uns, ob vielleicht dieser Umstand dadurch bedingt ist, dass die Hefe selbst Zucker enthalte?

Allein wir stellten Versuche mit lange Zeit hindurch auf dem Filter gewaschener Hefe an und erhielten ganz dieselben Resultate. Ferner suchten wir im Hefefiltrat Zucker nachzu-

1) Die Bezeichnungen 1 Theil Hefe und 20 Theile Hefe drücken überall nur das relative Verhältniss der Hefemengen in den einzelnen Versuchen aus. 
weisen, allein dieses gelang nicht, selbst wenn wir das Filtrat bis auf ein ganz kleines Volumen verdampften und dann die Trommer'sche Probe anstellten. Unsere Hefe enthielt also keinen Zucker.

Ausserdem schien es nicht wahrscheinlich die Gasblasen auf einen Gehalt der Hefe an Zucker zu beziehen; denn auch nach Zusatz von Natronlauge, wo sich die Gasräume allerdings verkleinerten, blieben noch immer verschieden grosse Gasblasen zurück, und zwar da, wo viel Hefe eine grosse Gasblase, und da wo wenig Hefe eine entsprechend kleine; - das wäre nicht der Fall gewesen, wenn jene Erscheinung vom Zucker herrührte, da, wie wir in unseren ersten Versuchen bereits bemerkt haben, bei einem verschiedenen Gehalt der Gährungssubstrate an Zucker die nach Natronzusatz zurückbleibenden Gasblasen gleich gross waren.

Wir waren auf diese Weise gezwungen anzunehmen, dass die Hefezellen an sich selbst in zuckerfreien Flüssigkeiten theilweise Zersetzungen in ihrem Leibe erleiden, wobei Kohlensäure und noch andere gasige Producte frei werden, da sich nur auf diesem Wege alle angeführten Erscheinungen gut erklären lassen.

Diese Annahme steht im Einklang mit der von Pasteur beobachteten, von Schützenberger und Destrem ${ }^{1}$ ) bestätigten, sogenannten secundären Gährung, welche die Hefezellen an sich selbst in indifferenten zuckerfreien Flüssigkeiten erleiden. -

Wie kommt es aber, dass in einer gekochten zuckerfreien Flüssigkeit die Gasblase um so viel kleiner wird?

Wir glauben dies dadurch zu rechtfertigen, dass die secundäre Gährung bei Gegenwart des in der absorbirten Luft enthaltenen Sauerstoffs viel lebhafter von Statten gehen dürfte, als in der gekochten, von Luft freien Flüssigkeit. Dass das Gasbläschen im gekochten Harn grösser war, als in gekochtem Wasser, rührt daher, dass der Harn ja überhaupt, wie wir bereits eruirt haben, ein besseres Mittel für den Gährungsprozess abgiebt als Wasser.

Aus dieser, „über die Erscheinung der Gasblase" eingeschal-

1) Schützenberger et Destrem, Compt. rend. Bd. 88. 288. 
teten Zwischenabhandlung haben wir zwei wichtige Thatsachen für unsere Arbeit zu verwerthen:

1) Gekochte zuckerfreie Flüssigkeiten Iiefern eine verhältnissmässig sehr kleine Gasblase.

2) Die Hefemenge hat einen Einfluss auf die Grösse der Blase, und kann dieselbe bei geringer Hefequantität gleichfalls klein werden. -

Wir wollen nunmehr im Folgenden sehen, ob wir aus diesen beiden Ergebnissen irgend welche Vortheile für unsere specielle Aufgabe, die Empfindlichkeit der Reaction zu steigern, gewinnen können.

Zunächst die erste Thatsache anlangend, so musste untersucht werden, ob nicht das Kochen, welches die secundäre Gährung beeinträchtigt, in einer zuckerhaltigen Flüssigkeit einen ebensolchen schädlichen Einfluss auf die primäre Gährung ausübte. Allein wir haben uns durch Versuche überzeugt, dass dem nicht so ist, sondern dass der Zucker in einer gekochten Flüssigkeit ebenso schnell und gut vergährt, wie in einer nicht gekochten, d. h. dieselbe Menge Kohlensäure bildet.

Wir hätten daher in dem Kochen ein Mittel, die Reactionsempfindlichkeit zu steigern.

Wir stellten nun, indem wir zuckerhaltigen und normalen Harn kochten und mit gekochtem Wasser auf das ursprüngliche Volumen wieder verdünnten, folgende Versuche an:

1. Den 3. Juni 1885. Harn, spec. Gew. 1009.

A. Harn ungekocht + Hefe.

B. Harn gekocht verdünnt + Hefe.

C. Harn ungekocht mit $\frac{1}{1} \sigma p \mathrm{Ct}$. Zuckergehalt + Hefe.

D. Harn gekocht verdünnt mit $\frac{1}{10}$ pCt. Zuckergehalt + Hefe.

Nach 24 Stunden. Bei A die ganze Kuppel des cylindrischen Robres vom Gase eingenommen; bei B ein grossstecknadelkopfgrosses Bläschen; bei $\mathrm{C} \frac{1}{1^{2}}$, bei D 3 $_{0}^{1}$ von der Flüssigkeit leer.

2. Den 4. Juni 1885. Harn, spec. Gew. 1010.

A. Harn ungekocht + Hefe.

B. Harn gekocht verdünnt + Hefe.

C. Harn ungekocht mit $\frac{1}{2} \sigma$ pCt. Zuckergehalt + Hefe.

D. Harn gekocht verdünnt mit $\frac{1}{z}$ pCt. Zuckergehalt + Hefe.

Bei $A$ und $C$ die Kuppel des cylindrischen Rohres von Flüssigkeit leer; bei B ein grossstecknadelkopfgrosses Bläschen, bei I) eine beinahe die Kuppel ausfüllende Blase. 


\section{9}

3. Den 5. Juni 1885. Harn, spec. Gew. 1022.

A. Harn gekocht verdünnt + Hefe.

B. - - - mit $\frac{1}{20}$ pCt. Zuckergehalt + Hefe.

C. Wasser gekocht + Hefe.

Nach 24 Stunden bei A ein grossstecknadelkopfgrosses B]äschen; bei $B$ eine mehr als linsengrosse Blase; bei $\mathrm{C}$ ein stecknadelkopfgrosses Bläschen.

4. Den 8. Juni 1885.

A. Wasser gekocht + Hefe.

B.

C. - - mit $\frac{1}{20}$ pCt. Zuckergehalt + Hefe.

D.

Nach 24 Stunden bei A und B ein stecknadelkopfgrosses Bläschen; bei $\mathrm{C}$ and $\mathrm{D}$ eine beinahe die ganze Kuppel einnehmende Blase.

Aus diesen Versuchen geht ohne Weiteres hervor, dass wir durch das Einführen des neuen Verfahrens noch $\frac{1}{20}$ pCt. Zucker im Harn sowohl wie im Wasser haben erkennen können.

Die Empfindlichkeit der Gährungsprobe wird also durch das Kochen bedeutend gesteigert.

Wie steht es nun mit der zweiten Thatsache, dass bei einer geringen Hefemenge das Gasbläschen im zuckerfreien Harn ebenfalls ganz klein ist? Könnten wir nicht auch auf diesem Wege die Empfindlichkeit der Reaction steigern, d. h. deutlicher ausgesprochene Differenzen zwischen kleine Quantitäten Zucker enthaltenden und zuckerfreien Harnen erhalten? -

Das wäre der Fall, wenn die geringe Hefemenge, welche im zuckerfreien Harn nur ein stecknadelkopfgrosses Bläschen erzeugt, dazu ausreichte den ganzen Zucker zur Vergährung zu bringen und also das Gasvolumen des zuckerhaltigen Harns nicht verkleinerte.

Folgender Versuch zeigt, dass dem nicht so ist. Und müssen wir demnach auf eine Steigerung der Reactionsempfindlichkeit durch kleine Hefenquantitäten Verzicht leisten.

Den 14. Juni 1885. Harn, spec. Gew. 1009.

A. Harn +1 Theil Hefe.

B. $\quad-\quad+\frac{1}{10}$

O. - mit $\frac{1}{10}$ pCt. Zuckergehalt +1 Theil Hefe.

D.

Nach 24 Stunden bei A die Kuppel des cylindrischen Robres vom Gas eingenommen; bei $\mathrm{B}$ ein stecknadelkopfgrosses Bläschen; bei C der Gasraum reicht $2 \rightarrow 3 \mathrm{~mm}$ über die Kuppel hinaus, bei $\mathrm{D}$ ein linsengrosses Bläschen. 
Indem wir uns vorbehalten später noch auf den Einfluss der Hefemenge zurückzukommen, wollen wir uns wieder zum Kochverfahren wenden.

Bisher hatten wir den Harn stets, um ihn in seiner Concentration nicht zu ändern, nach dem Kochen mit gekochtem Wasser auf das ursprüngliche Volumen wieder verdünnt.

Es fragt sich, ob diese Manipulation wirklich nöthig ist, d. h. ob nicht der gekochte unverdünnte, also etwas concentrirtere, Harn die Reaction ebenso deutlich hervorzubringen im Stande ist?

Um diese Frage zu beantworten, mussten wir studiren, welchen Einfluss die Concentration des Harns auf die Gährung ausübt und stellten folgende Versuche zunächst mit ungekochtem, dann mit gekochtem verdünntem und unverdünntem Harn an:

1. Den 15. Juni $188 \overline{5}$.

A. Harn nicht gekocht (spec. Gew. 1009) + Hefe.

B. - - - - - - mit $\frac{1}{10}$ pCt. Zuckergehalt + Hefe.

C. - - - - (spec. Gew. 1023) + Hefe.

D. - - - - - - mit $\frac{1}{10}$ pCt. Zucker + Hefe.

Nach 24 Stunden bei $\mathrm{C}$ und $\mathrm{D}$ entsprechend grössere Gasräume, wie bei $\mathrm{A}$ and $\mathrm{B}$.

2. Den 9. Juni 1885. Harn, spec. Gew. 1012.
A. Harn gekocht unverdünnt + Hefe.
B. - - verdünnt
C. - - unverdünnt mit $\frac{1}{10}$ pCt. Zuckergehalt + Hefe.
D. - - verdünnt
E. - - unverdünnt $-\frac{1}{20} \mathrm{pCt}$.
F. - verdünnt

Nach 24 Stunden bei A ein linsengrosses Bläschen, bei B ein grossstecknadelkopfgrosses Bläschen; bei $\mathrm{C}$ und $\mathrm{E}$ entsprechend grössere Gasräume, als bei $D$ ) und $F$.

3. Den 10. Juni 1885. Harn, spec. Gew. 1022.

A. Harn gekocht unverdünnt + Hefe.

B. - - verdünnt

C. - - unverdünnt mit $\frac{1}{20}$ pCt. Zuckergehalt + Hefe.

D. - - verdünnt

[Das Einkochen geschab diesmal bis auf weniger als die Hälfte des ursprünglichen Volumens, während wir im vorigen Versuch das Kochen nur bis auf $\frac{3}{4}$ des Volumens vorgehen liessen.]

Nach 24 Stunden: Bei A ist das Blässhen nur etwas grösser als bei B; bei $\mathrm{C}$ ist der Gasraum um ein Wenig grösser, als bei $\mathrm{D}$. 


\section{1}

Alle diese Versuche zeigen deutlich den günstigen Einfluss, den die Concentration des Harns auf die Gährung ausübt, zwar erstreckt sich dieser günstige Einfluss auch auf die secundüre Gährung, gleichwohl ist das Gasbläschen im geknchten concentrirten zuckerfreien Harn nur um einen geringen Bruchtheil grösser, als im gekochten verdünnten Harn und beeinträchtigt nicht die Deutlichkeit der Zuckerreaction.

Somit wäre das Vordünnen des Harns nach dem Kochen nicht nothwendig, und wir werden daher in unseren noch folgenden Versuchen mit gekochtem unverdünntem Harn arbeiten.

In allen unseren Versuchen pflegten wir stets ziemlich gleich grosse Hefemengen (etwa ein klein haselnussgrosses Stïck Presshefe auf $10 \mathrm{ccm}$ Flüssigkeit) zu nehmen, ohne dass wir auf diesen Umstand einen grossen Werth legten. Nachdem wir aber, wie bereits oben angegeben, erkannt haben, dass die Hefemenge in Bezug auf den Gährungsvorgang nicht gleichgültiger Natur ist, schien es uns von Interesse, das Verhalten der Hefemenge genauer zu untersuchen.

Folgende Versuche wurden in der Richtung angestellt:

1. Den 12. Juni 1885. Harn, spec. Gew. 1022.

A. Harn gekocht + ein haselnussgrosses Stück Hefe.

B. - - $\quad+\frac{1}{10}$ Theil davon.

C. - - mit $\frac{1}{10} \mathrm{pCt}$. Zuckergehalt + ein haselnussgrosses Stück Hefe.

D. - $\quad$ - $\quad$ - $\quad-\quad+1 \frac{1}{10}$ Theil daron.

Nach 24 Stunden bei A ein linsengrosses Gasbläschen; bei B ein kleinstecknadelkopfgrosses Gasbläschen; bei $\mathrm{C} \frac{1}{12}$ des cylindrischen Robres von F]üssigkeit leer, bei $\mathrm{D}$ eine kleinknopfgrosse Blase.

2. Den 13. Juni 1885. Harn, spec. Gew. 1018.

A. Harn gekocht + ein haselnussgrosses Stück Hefe.

B. - - mit $\frac{1}{10}$ pCt. Zuckergehalt + ein haselnussgrosses Stück Hefe.

C. - - - $-\frac{1}{20}$ pCt.

D. - $\quad-\quad+\frac{1}{10}$ Theil davon.

E. - - mit $\frac{1}{10}$ pCt. Zuckergehalt $+\frac{1}{10}$ Theil davon.

F. - - $\quad-\frac{1}{30} \mathrm{pCt}$.

(Der Harn wurde 10 Minuten lang gekocht.)

Nach 24 Stunden bei A ein linsengrosses Bläschen, bej $\mathrm{B} \frac{1}{12}$ des cylindrischen Rohres von der Flüssigkeit leer, bej $\mathrm{C}$ ausser der Kuppe noch 1 bis $2 \mathrm{~mm}$ des graden Theils mit Gas gefüllt; - bei D ein kleinstecknadelkopfgrosses Bläschen, bei E eine grosslinsengrosse Blase, bei $\mathrm{F}$ ein linsengrosses Bläseben. 
3. Den 18. Juni 1885. Harn, spec. Gew. 1021,

Alles 10 Minuten gekocht unverdünnt.

A. Harn gekocht $+\mathbf{1} \mathrm{g}$ Hefe.

B. - - $\quad+\frac{1}{4} g$ -

C. - - $\quad+\frac{1}{8} \mathrm{~g}-$

D. - - mit $\frac{1}{20}$ pCt. Zuckergehalt $+1 \mathrm{~g}$ Hefe.

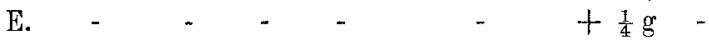

F. - - - - - $\quad-\quad-\quad+\frac{1}{8} \mathrm{~g} \quad-$

Nach 24 Stunden. (Wir haben diesmal, da es mit der Beschreibung der Gasblasen eine missliche Sache ist, die Durchmesser der entstandenen Gasblasen gemessen und geben die Zahlen an.) Bei A $5 \mathrm{~mm}, \mathrm{~B} 3 \mathrm{~mm}, \mathrm{C}$ $2 \frac{1}{2} \mathrm{~mm}$, D $12 \mathrm{~mm}$ (der Gasraum ragt $1-2 \mathrm{~mm}$ über die Kuppel hinaus), E $7 \frac{1}{4} \mathrm{~mm}, \mathrm{~F} 5 \mathrm{~mm}$.

Aus Versuch 2 folgt, dass ein und dieselbe Quantität Hefe in einer concentrirten Zuckerlösung mehr zur Vergährung bringt, als in einer weniger concentrirten; aus Versuch 1, 2 und 3 , dass in ein und derselben Zuckerlösung wenig Hefe, weniger Zucker zur Vergährung bringt, als viel Hefe (bis $1: 10$ ).

[Die grösste Hefemenge, welche wir angewandt haben, war nehmlich ungefähr 1 auf 10 Flüssigkeit, nimmt man noch mehr, so wird die Vergährung dadurch nicht erheblich gesteigert.]

Aus allen diesen Versuchen geht jedenfalls hervor, dass man bei Anstellung der Gährungsprobe - wobei man stets einen normalen Controlharn haben muss, den man in Gemeinschaft mit dem zuckerhaltigen Harn ein und derselben Behandlung unterwirft - immer gleiche Mengen Hefe für beide nehmen muss, da man sonst in dem, vielleicht mit einer grossen Hefequantität versetzten, Normalharn einen grösseren Gasraum erhalten könnte, als in dem, mit sehr wenig Hefe behandelten, geringe Mengen Zucker enthaltenden, Harn, und man auf diese Weise leicht Täuschungen unterliegt.

Wie aus unserer ganzen Arbeit ersichtlich, beurtheilen wir die Frage, ob in einem Harn Zucker enthalten sei, oder nicht, nur nach dem Verhalten seines, bei der Gährung entstandenen, Gasvolumens zu dem ebenso behandelten Controlharn. Der Nachweis von ein wenig Kohlensäure allein in dem Gasvolumen kann uns keineswegs davon überzeugen, dass der Harn Zucker enthäIt, denn wir haben gesehen, dass die secundäre Gährung der Hefe gleichfalls Kohlensäure liefert. 
Wir versuchten das zweite Product des zerfallenen Zuckers zu diesem Behufe zu verwerthen, nehmlich den Alkohol; wir führten den Nachweis desselben indem wir den vergohrenen zuckerhaltigen Harn filtrirten, das Filtrat destillirten und das erste Destillationsproduct mit Jod and Natronlauge versetzten; beim Erwärmen entstand Jodoformgeruch und beim Erkalten schieden sich die charakteristischen Jodoformkrystalle aus.

Allein der Nachweis von Alkohol genügt ebensowenig um Zucker zu zeigen, wie der von Kohlensäure. Wir haben nehmlich auch im Filtrat des zuckerfreien Harns und Wassers der Gährungsröhrchen denselben Nachweis von Alkohol führen können. Dieser rührt gleichfalls ebenso wie die Kohlensäure von der secundären Gährung her.

So ist man denn beim qualitativen Nachweis von Zucker ausschliesslich auf die Differenz der Gasvolumina angewiesen.

Recapituliren wir, so hätten wir kurz Folgendes als Resumé unserer Arbeit zu betrachten:

1) Der Harn giebt günstigere Bedingungen für die Gährung $a b$, als Wasser, und zwar übt die Concentration desselben einen günstigen Einfluss aus.

2) Zusätze vom Salzen oder anderen Substanzen sind bei Anstellung der Gährungsprobe nicht von Nöthen.

3) Man kann vermittelst der Gährungsprobe ohne Weiteres, wenn man gleiche Hefequantitäten nimmt, noch $\frac{1}{10} \mathrm{pCt}$. Zucker erkennen.

4) Durch 10 Minuten langes Kochen des Harns gelingt es sogar noch $\frac{1}{20}$ pCt. Zucker nachzuweisen.

5) Beweisend für Zucker ist nur eine mehr oder weniger beträchtliche Differenz zwischen dem grösseren Gasvolumen des Untersuchungsharns zu dem kleineren Gasvolumen (Gasblase) des normalen, in genau derselben Weise behandelten, Controlharns. Der Nachweis von Kohlensäure allein im entstandenen Gase des Untersuchungsharns beweist noch nicht Zucker und ebensowenig genügt der Nachweis von Alkohol resp. Jodoform bildender Substanz im gegohrenen Harn hierzu, wo es sich um kleine Quantitäten Zucker handelt. 
Im Anschluss an die von uns eben abgehandelte Gährungsprobe haben wir noch eine Methode geprüft, welche es ermöglicht, einen sicheren Nachweis von Zucker zu führen.

Wir meinen das von E. Salkowski') angegebene Verfahren, welches in einer Ausfällung des Zuckers mit Kupferoxydhydrat besteht; man ist auf diese Weise im Stande den Zucker allein, isolirt von anderen Harnbestandtheilen zu erhalten und einer Prüfung zu unterwerfen. Das Verfahren wird nach demselben Autor zweckmässig folgendermaassen ausgeführt: $20 \mathrm{ccm}$ Harn werden mit $10 \mathrm{ccm}$ Kupferlösung (199,52 krystallisirter Kupfervitriol zu 1 Liter gelöst) und 17,6 Normalnatronlauge versetzt, gut durchgeschïttelt und etwa 20-25 Minuten stehen gelassen, dann $100 \mathrm{ccm}$ Wasser hinzugesetzt, nach starkem Umschütteln durch ein grosses Faltenfilter filtrirt. Der Niederschlag wird sodann, indem das Filter mit dem Glasstab durchstochen wird, mit der Spritzflasche von demselben in ein reines Becherglas abgespült; nun wird Schwefelwasserstoff eingeleitet, bis alles gelöste Kupfer wieder ausgefallen ist, filtrirt und das Filtrat bis auf $20 \mathrm{ccm}$ eingedampft. Mit diesem erhaltenen Reste stellt man nun die Trommer'sche Probe an.

Wir haben nun, um die Empfindlichkeit dieses Verfahrens zu constatiren, folgende Versuche angestellt.

1. Harn mit $\frac{1}{2}$ pCt. Zuckergehalt.

2. $\quad-\quad-\frac{1}{4} \mathrm{pCt}$.

3., - $\quad-\frac{1}{10} \mathrm{pCt}$.

In diesen Versuchen trat in dem zuletzt erhaltenen verdampften Filtrat die Trommer'sche Probe gleich beim Erwärmen ein; ebenso die von N ylander modificirte Böttger'sche.

4. Harn mit $\frac{1}{20}$ pCt. Zuckergehalt.

In dem verdampften Filtrat trat die Trommer'sche Probe beim Erkalten ein.

$\overline{0}$. Normaler Harn, nach derselben Methode behandelt.

Im Filtrat erhielten wir durch die Trommer'sche Probe keine Reduction.

6. Harn mit $\frac{1}{20}$ pCt. Zuckergehalt.

7. Normaler Harn.

In 6 und 7 stellten wir zunächst mit den rohen Harnen die Trommer'sche sowohl, wie die modificirte Böttger'sche Probe an, vermochten aber weder durch die eine noch durch die andere eine Reduction za bekommen;

1) Salkowski und Leube, Die Lehre vom Harn. S. 223. Zeitschr. f. phys. Chem. Bd. III. S. 96. 
ja die Böttger'sche Probe ergab sogar im normalen Harn (7) einen etwas graubräunlichen, im zuckerhaltigen (6) einen weissen Niederschlag mit einem Stich in's Gelbe.

Wir behandelten nun beide Harne nach dem angegebenen Verfahren und stellten mit dem verdampften Filtrat die Trommer'sche Probe an.

In 6 , also dem $\frac{1}{20}$ pCt. Zucker enthaltenden Harn, verschwand schon beim gelinden Erwärmen die blaue Farbe, sie ward strohgelb; als die Flïssigkeit zu kochen anfing, wurde sie von der Flamme entfernt; schon nach wenigen Secunden trat eine citronengelbe Trübung ein, welche immer mehr zunahm; am Boden des Reagensglases bemerkt man nach einigen Minuten einen mehr oder weniger orangerothen Niederschlag mit einem Stich in's Gelbe.

Im Gegensatz dazu nahm das eingedampfte Filtrat von 7, also normalem Harn, ebenso behandelt, beim Erwärmen nicht jene strohgelbe Farbe au; beim Erkalten blieb noch die blaue Farbe zurück; es bildete sich (zwar) nach einiger Zeit ein Bodensatz im Reagensglase; dieser sab schmutzig grau aus.

Diese Versuchsreihe zeigt also, dass man vermittelst dieses Salkowski'schen Verfahrens noch im Stande ist selbst $\frac{1}{20} \mathrm{pCt}$. Zucker sicher nachzuweisen, wie ich nachträglich bemerke, ist E. Salkowski zum selben Ergebniss gekommen.

Es scheint demnach dieses Verfahren zum Nachweis von kleinen Mengen Zucker, die Empfindlichkeit und Sicherheit anlangend, der Gährungsprobe gleichgestellt werden zu können. Es werden in dieser Hinsicht noch Versuche mit verschiedenen Harnen sehr wünschenswerth, doch war ich durch äussere Verhältnisse leider gezwungen, die Versuche abzubrechen.

Zum Schluss sei es mir gestattet, Herrn Professor Dr. Salkowski für die Anregung zu dieser Arbeit sowie für die wohlwollende mir zu Theil gewordene Unterstützung den innigsten Dank auszusprechen. 